\title{
Social Power as an Exchangeable Resource for Distributed Multi-Agent Systems
}

\author{
Don Hayes \\ Department of Computer Engineering and \\ Computer Science \\ University of Arkansas \\ Fayetteville, AR 72701, USA. \\ dlh05@uark.edu
}

\author{
Henry Hexmoor \\ Department of Computer Engineering and \\ Computer Science \\ University of Arkansas \\ Fayetteville, AR 72701, USA. \\ hexmoor@uark.edu
}

\begin{abstract}
An innovative model of structuring agent control based on the implicit concept of transferring a portion of social power upon a series of requests for delegation being offered. Existing models are examined and evaluated. We present the concept of social power as a resource that is exchanged within an agent group instead of a static power structure or one based on dynamic rank. Every time an agent commands another agent, the power structure within the agent group is redistributed. Providing intuitive validation, our simulation results for a single agent using our model of power generation and decay are explored.
\end{abstract}

KEYWORDS: Multi-Agent Systems, Social Power, Power, Tokens, Fault-Tolerance

\section{INTRODUCTION}

In distributed multi-agent systems there are a multitude of techniques used to organize the power structure of the agent community. The reasons for using a distributed model instead of a centralized model stem from issues of performance. The performance of centralized systems does not scale as the number of agents increases [1]. BenAmi and Shehory do not address the concept of social power. Techniques have been proposed for generating agent-organized social networks based on physical structure or agent performance; however the techniques do not address balancing social power [2]. In order to design a distributed system that performs well, we rely on the concept of tokens. Tokens are unique references for resources, information, and assignable tasks; though items the tokens reference do not need to be unique [5]. This model's fault-tolerance was not examined, but due to the static nature of the tokens it would be at risk if an agent fails, is destroyed, or separated from the community while in possession of a token. Sekiyama and Okade present a system for cooperation in an environment consisting of directed agents and subjective distance between agents. The model has metrics for determining the level of cooperation in a the system; however, the distribution of power is uniform and is not able to be redistributed [4]. Definitions of power and how its relative value is influenced in a group of agents is explored; but, the ability of agents to change in absolute levels of power is not [3].

We propose a new model in order to address the issues of autonomy, fault-tolerance, and a power structure that can change during execution. In this model, agents that wish to influence other agents in order to increase the utility of the community or of themselves must initiate a trade of resources which will reciprocally allow the commanded agent to influence others. These resources will have a limited lifespan and be continually regenerated in order to maintain fault-tolerance. The heuristics used to determine agent/group/community utility are beyond the scope of this paper, as is the issue of deception. We define an agent community to be all agents with shared goals that are potentially able to communicate with one another. A group is any collection of agents that is currently capable of communicating with one another. Social power is defined as the ability of a particular agent to exert leadership and direct the actions other agents.

To influence other agents, an agent must transfer a portion of social power to the agent being commanded. This resource will be called a Power Token and PT will be used for brevity. In order for an agent $a$ to direct agent $b$, some number of PTs must be transferred from $a$ to $b$. If $b$ does not wish to obey the directive given by $a$, agent $b$ may choose to give agent $a$ a greater number of PTs. In other words, if $a$ gives $b$ a command with $n \mathrm{PTs}, b$ has the option of refusing by giving $n+1$ PTs to agent $a$. This bidding ensures that the more strongly two agents feel about opposing goals or tasks, the greater the power structure will change. 
The amount of PTs probabilistically decay based on the current number of PTs an agent possesses. If an agent has a large amount of power, it is likely that some of that power will decay. This probability is termed the decay rate. Agents with little or no power have a probability to generate a new PT, termed the generation rate. The total number of PTs in the system can be adjusted by altering the decay rate and the generation rate. The values must be set such that the average number of PTs in the system is stable. This will allow an isolated group to have a similar ratio of power to the community as a whole.

The thought processes and rationale motivating this system are as follows. Agents should share information when feasible to allow agents to plan and act better; though how this planning and acting is performed is beyond our scope. Power should be distributed among agents and the power structure should continually mutate. Continually altering the power structure will likely result in less efficient behavior, however it will ensure that any errors introduced from conclusions on incomplete information will be contained. The following conditions allow any group of agents to be separated from the community and rejoin without permanently impacting the overall power level of the community. Power tokens should decay if they are in excess. Agents should generate power if they have an amount below the intended value.

\section{EFFECT ON AUTONOMY}

An agent with a large amount of power relative to other agents in the group will have a high amount of autonomy. An agent will gain power after it has had its autonomy infringed upon by another agent. By accepting a directive, an agent will gain power to use in the future. An agent will accept a directive because of agreement with the directive or because the agent does not posses enough PTs to make a counter-offer. A miserly or greedy agent that hoards power will not gain large excesses of power due to the decay rate of PTs. As the number of PTs an agent possesses increases, the probability of a PT decaying increases. An agent that has not carried out any directives, and consequently not received power for some amount of time, will generate a new PT. This generation is governed by the generation rate. This ensures that any group of agents that is isolated from the community may continue working toward the goals of the community. With a stable flow of PTs this ensures that any group may be seamlessly brought back into the community without a need to adjust the power distribution of the group or the community. Agents should be encouraged to spend PTs because they expire. This keeps the power of the system as a whole stable, yet transient. Power transfers between agents are zero-sum; though transient totals of power in the system may be slightly above or slightly below the expected total.

In order to change the autonomy of agents in the system, the ratio of PTs to agents must be altered. A system with a high proportion of PTs to agents will lead to higher individual autonomy, as agents are more able to refuse commands given to them. A system with a low proportion of power to agents will have low autonomy for individual agents, because agents will not have the power necessary to refuse commands. Changing the ratio of PTs during run-time by altering the decay rate and generation rate is not explored.

\section{AN EXAMPLE USING UAVS}

Suppose we have a community of 100 Unmanned Ariel Vehicles (UAVs), each distinctly controlled by an agent. In this example the terms agent and UAV are used interchangeably. Assume we have a 1:1 ratio of PTs to agents. This gives $100 \mathrm{PTs}$ in the system. We set the decay rate and generation rate such that the overall power is stable. Consider an isolated group $M$ of 10 agents. A group is considered isolated if the only contact any agent has is with members of the same group. After a sufficient amount of time, $M$ will have $10 \mathrm{PTs}$, since we set our ratio at $1: 1$ and the group has 10 agents. In a similar manner, the remaining 90 agents will have decayed their total PTs to 90. Continuing the UAV example, these isolated agents might be pinned down by enemy fire, or simply out of communication range. The generation of new PTs by the isolated group allows the isolated group to re-organize its power structure without the need for contact from the community as a whole. When the group is reunited with the community, no further power adjustments will need to be made, as power is already balanced at a total of 100 agents and 100 PTs.

Suppose a single UAV with 10 PTs is eliminated. The system now has 99 agents and 90 PTs. This situation is similar to the previous example. The proportion of PTs to agents in the community is less than the set ratio. The community will generate new PTs and stabilize to a total of 99 PTs after some amount of time.

Agent Don wants agent Henry to examine an area, to find out if it is safe for passage. Don has one PT and Henry has zero. Don gives one PT to Henry with the order to examine the specified area. A single PT is transferred from Don to Henry and Henry must examine the area. Time passes and Henry receives another PT, bringing Henry's total PTs up to two. Don has generated a new PT and wants Henry to act as an escort for Don's flight. Don offers one PT to Henry, and Henry does not want to act as an escort right now, so counter-offers with two PTs. 
Because Don has only one PT, Don has no choice but to accept this counter offer, receiving two PTs from Henry. More time passes and two of Don's PTs expire, the ending state leaves Don with one PT and Henry with zero. The total power of both agents in this example peaks at three, and stabilizes at one. In this particular example the starting value is the stable value, though this is not required. After each transaction the power structure changed.

\section{IMPLEMENTATION}

The value of the decay rate is prescribed to be the probability rate at which power decreases. The generation rate is likewise prescribed to be the probability rate at which the current amount of power the agent possesses increases. A probabilistic generation causes the absolute amount of power to increase when there is a shortage; this is the desired behavior in order to maintain the ratio of power to agents. The probability of generating new power is equal to $e$ raised to the power of negative one multiplied by the generation rate multiplied by the current power.

$$
G(p)=e^{\left(-\lambda G^{*} p\right)}
$$

As the value of current power approaches zero, the exponential, and likewise the probability of generating new power, will approach one.

Another property of this model is that for an isolated group, power will generate or decay in order to maintain the same ratio of power to agents as the community of its origin. Using a probabilistic decay rate, in every unit of time there is a probability of decaying existing power based on the current amount of power. For higher amounts of power, the likelihood of decay is larger. The rate of decay increases because the probability of decay is equal to one minus $e$ raised to the power of negative one multiplied by decay rate multiplied by the current power.

$$
D(p)=1-e^{\left(\lambda{ }^{*} p\right)}
$$

For large values of current power, the exponent is large in magnitude and negative, this causes the exponential term to approach zero and the probability to approach one. Absolute power in excess of the prescribed amount decays over time. This is the desired behavior to maintain the specified ratio.

\footnotetext{
$\mathrm{p}$ is the current amount of power (PT) for a particular agent. $\lambda_{\mathrm{G}}$ is inversely proportional to the the
}

rate of generation for new power. $\lambda_{\mathrm{D}}$ is directly proportional to the the rate of decay for existing power.

$$
\begin{aligned}
& \left.\mathrm{G}(\mathrm{p})=\mathrm{e}^{(-\lambda} \mathrm{G}^{*} \mathrm{p}\right) \\
& \left.\mathrm{D}(\mathrm{p})=1-\mathrm{e}^{(\lambda}{ }^{*} \mathrm{p}\right) \\
& \operatorname{Total}(\mathrm{p}+1)=\operatorname{Total}(\mathrm{p})+\mathrm{G}(\mathrm{p})-\mathrm{D}(\mathrm{p})
\end{aligned}
$$

Each agent uses the following. The power for the next state is equal to the power for the current state plus the probability of generating new power minus the probability of decaying existing power.

$$
\operatorname{Total}(p+1)=\operatorname{Total}(p)+G(p)-D(p)
$$

Agents do not have or need any knowledge about other agents. When generation occurs, the total power is incremented by one. Decay operates in a analogous fashion; total power is decremented by one when decay occurs. Generation and decay occur with a probability given by $G(p)$ and $D(p)$.

\section{SIMULATION}

We have implemented an iterative process for modeling power distribution among a community of similar agents. Each trial was attempted for 1000 iterations. Each trial set consisted of 50 trials. In the first set of trials (figure 1), $\lambda_{\mathrm{G}}$ and $\lambda_{\mathrm{D}}$ are set to 0.1 . For initial power values of 0 , 10 , and 50 the final power stabilizes at 6.8. As the initial value approaches the number of iterations, the culminating power increases; this is because the power does not have the opportunity to decay before the iterations cease. The second set of trials consists of setting the initial population to 10 , and varying $\lambda_{\mathrm{G}}$ and $\lambda_{\mathrm{D}}$. As $\lambda_{\mathrm{G}}$ gets larger, the final power decreases. For small values of $\lambda_{D}$ the final power increases by a large proportion.

These results are for a single agent. Agents determine their own power independently, based only on their current power. Since results are independent for each agent, this will scale in the same fashion for multiple agents. We illustrate that the culminating stable power for an agent will be independent of its current power. This allows agents to enter and leave the community without affecting the power of the group. 


\begin{tabular}{|c|c|}
\hline Initial Power & Final Power \\
\hline 0 & 6.96 \\
\hline 10 & 6.80 \\
\hline 50 & 6.86 \\
\hline 100 & 7.06 \\
\hline 500 & 7.30 \\
\hline 1000 & 10.60 \\
\hline
\end{tabular}

Figure 1. Varying Initial Power

\begin{tabular}{|r|r|r|}
\hline$\lambda_{G}$ & $\boldsymbol{\lambda}_{\mathbf{D}}$ & Final Power \\
\hline .1 & .1 & 7.02 \\
\hline .1 & .5 & 3.12 \\
\hline .1 & .9 & 2.14 \\
\hline .5 & .1 & 2.98 \\
\hline .5 & .5 & 1.54 \\
\hline .5 & .9 & 1.24 \\
\hline .9 & .1 & 2.02 \\
\hline .9 & .5 & 1.12 \\
\hline .9 & .9 & .98 \\
\hline
\end{tabular}

Figure 2. Varying Growth and Decay

\section{CONCLUSIONS}

We have introduced the concept of power as a resource that can be bartered with and transfered. An individual agent may control its own autonomy by utilizing this power resource to avoid submitting to a directive. An agent may also direct other agents, and gain either cooperation or additional power. The autonomy of a group or community of agents may be purposefully steered via altering the power to agent ratio. A large ratio of power to agents leads to increased autonomy. Using UAVs as a scenario, we examined the feasibility of our power model for developing a control tool for manipulating individual autonomy. Specific implementation issues were discussed. Simulation results were offered. By choosing appropriate values for the generation rate and the decay rate, desired average power can be attained. Agents compute their own power independently, removing the need for communication. The power of an agent, and by extension any sub-group of agents will converge toward a known value, granting robustness to the system.

\section{REFERENCES}

[1] Ben-Ami D. \& O. Shehory, "A Comparative Evaluation of Agent Location Mechanisms in Large Scale MAS," AAMAS'05, Utrecht, Netherlands, 2005, pp. 339-346.

[2] Gastion M. \& M. desJardins, "Agent-Organized Networks for Dynamic Team Formation," AAMAS'05, Utrecht, Netherlands, 2005, pp. 230-237.

[3] Hexmoor H., "Absolute Model of Autonomy and Power: Toward Group Effects," The AAAI-02 Workshop on Autonomy, Delegation, and Control: Interacting with Autonomous Agents, Edmonton, Canada, 2002, pp. 323-334.

[4] Sekiyama K. \& Y. Okade, "Formation of Cooperation Structure by Interaction Network in Directed Multi-Agent," AAMAS'05, Utrecht, Netherlands, 2005, pp. 207-213.

[5] Xu Y., P. Scerri, B. Yu, S. Okamoto, M. Lewis, K. Sycara, "An Integrated Token-Based Algorithm for Scalable Coordination," AAMAS'05, Utrecht, Netherlands, 2005, pp. 407-414. 